\title{
TGFBR2-dependent alterations of microRNA profiles in extracellular vesicles and parental colorectal cancer cells
}

\author{
FABIA FRICKE $^{1,2^{*}}$, VERONIKA MUSSACK $^{3 *}$, DOMINIK BUSCHMANN $^{3}$, INGRID HAUSSER $^{4}$, \\ MICHAEL W. PFAFFL ${ }^{3}$, JÜRGEN KOPITZ ${ }^{1,2}$ and JOHANNES GEBERT ${ }^{1,2}$ \\ ${ }^{1}$ Department of Applied Tumor Biology, Institute of Pathology, \\ Heidelberg University Hospital, D-69120 Heidelberg; ${ }^{2}$ Clinical Cooperation Unit Applied Tumor Biology, \\ German Cancer Research Centre (DKFZ), D-69120 Heidelberg; ${ }^{3}$ Department of Animal Physiology and Immunology, \\ TUM School of Life Sciences Weihenstephan, Technical University of Munich, D-85354 Freising; \\ ${ }^{4}$ EM-Lab, Institute of Pathology, Heidelberg University Hospital, D-69120 Heidelberg, Germany
}

Received April 15, 2019; Accepted July 22, 2019

DOI: 10.3892/ijo.2019.4859

\begin{abstract}
In colorectal cancer (CRC) with microsatellite instability (MSI), $>90 \%$ of cases are affected by inactivating frameshift mutations of transforming growth factor $\beta$ receptor type 2 (TGFBR2). TGFBR2 deficiency is considered to drive MSI tumor progression by abrogating downstream TGF- $\beta$ signaling. This pathway can alter the expression of coding and non-coding RNAs, including microRNAs (miRNAs), which are also present in extracellular vesicles (EVs) as post-transcriptional modulators of gene expression. In our previous study, it was shown that TGFBR2 deficiency alters the protein composition
\end{abstract}

Correspondence to: Dr Johannes Gebert or Ms. Fabia Fricke, Department of Applied Tumor Biology, Institute of Pathology, Heidelberg University Hospital, Im Neuenheimer Feld 224, D-69120 Heidelberg, Germany

E-mail: johannes.gebert@med.uni-heideberg.de

E-mail: f.fricke@dkfz.de

*Contributed equally

Abbreviations: p-adj, adjusted P-value; CRC, colorectal cancer; $\mathrm{Cq}$, quantification cycle; DGE, differential gene expression; MMR, mismatch-repair; dox, doxycycline; dT, TGFBR2 deficient; EMT, epithelial-to-mesenchymal transition; EV, extracellular vesicle; FU, fluorescent unit; HCA, hierarchical cluster analysis; $\log 2 \mathrm{FC}, \log 2$ fold change; miRNA, microRNA; MSI, microsatellite instability; MSS, microsatellite stability; NTA, nanoparticle tracking analysis; PCA, principal component analysis; pT, TGFBR2 proficient; rRNA, ribosomal RNA; RT-qPCR, reverse transcription-quantitative polymerase chain reaction; RNA-Seq, RNA sequencing; snoRNA, small nucleolar RNA; snRNA, small nuclear RNA; TEM, transmission electron microcopy; TGFBR2, transforming growth factor- $\beta$ receptor type 2; TME, tumor microenvironment; tRNA, transfer RNA

Key words: extracellular vesicles, exosomes, microRNA, small RNA sequencing, transforming growth factor $\beta$ receptor type 2 , DNA mismatch repair deficiency, microsatellite instability, colorectal cancer and function of EVs in MSI tumors. To investigate whether mutant TGFBR2 may also affect the miRNA cargo of EVs, the present study characterized miRNAs in EVs and their parental MSI tumor cells that differed only in TGFBR2 expression status. The HCT116-TGFBR2 MSI cell line model enables the doxycycline (dox)-inducible reconstituted expression of TGFBR2 in an isogenic background (-dox, TGFBR2 deficient; +dox, TGFBR2 proficient). Small RNA sequencing of cellular and EV miRNAs showed that the majority of the miRNAs (263/471; 56\%) were shared between MSI tumor cells and their EVs. Exploratory data analysis revealed the TGBFR2-dependent cluster separation of miRNA profiles in EVs and MSI tumor cells. This segregation appeared to result from two subsets of miRNAs, the expression of which were regulated in a TGFBR2-dependent manner (EVs: $n=10$; MSI cells: $n=15$ ). In the EV subset, 7/10 miRNAs were downregulated and 3/10 were upregulated by TGFBR 2 deficiency. In the cellular subset, 13/15 miRNAs were downregulated and 2/15 miRNAs were upregulated in the TGFBR2-deficient cells. The present study emphasizes the general overlap of miRNA profiles in MSI tumor cells and their EVs, but also highlights the impact of a single tumor driver mutation on the expression of individual miRNAs, as exemplified by the downregulation of miR-381-3p in TGFBR2-deficient MSI tumor cells and their secreted EVs.

\section{Introduction}

In colorectal cancer (CRC), a subset is characterized by the loss of DNA mismatch repair (MMR) function, leading to high frameshift mutation rates, predominately at repetitive DNA sequences (microsatellites). The resulting phenotype manifests as microsatellite instability (MSI) (1). A large number of genes are affected by microsatellite instability, however, only some of them are considered to be drivers of MSI cancer. One of the most frequent MSI tumor driver mutations occurs in a coding polyadenine (A10) tract in exon 3 of the transforming growth factor $\beta$ receptor 2 (TGFBR2) gene $(2,3)$. It is reported that $>90 \%$ of MSI tumors are affected by frameshift mutations in the TGFBR2 gene, leading to impaired receptor expression and abrogated downstream signaling (3). The TGFBR2 protein is 
a transmembrane serine-threonine kinase and serves as the primary receptor for non-canonical and canonical TGF- $\beta$ signaling. In the canonical pathway, binding of the TGF- $\beta 1$ ligand induces the dimerization of TGFBR2 and TGFBR1, leading to Smad-dependent intracellular signal propagation. Receptor-associated Smad proteins, such as Smad2 and Smad3, are then phosphorylated, allowing complex formation with Smad4. The functional complex translocates into the nucleus, where it interacts with different transcription factors to control expression of several target genes and proteins that are crucial for maintenance of the colonic epithelium (4). As downstream mediators of TGFBR2 signaling, Smad proteins can also affect the processing and expression of non-coding RNAs, including microRNAs (miRNAs) (5). With an average size of 22 nucleotides, miRNAs provide fine-tuning of protein abundance by silencing target gene expression at the post-transcriptional level $(6,7)$. Approximately $60 \%$ of protein-coding genes are regulated by miRNAs, which therefore play a key role in maintaining cellular homeostasis (8).

As important regulators of numerous normal and pathological processes, including colorectal tumorigenesis, functional miRNAs, among other molecules, have been identified in the cargo of extracellular vesicles (EVs) (9-12). Following transfer to cells in the microenvironment or at more distant sites, the functional cargo of EVs can elicit biological responses in recipient cells (13). As the EV cargo composition reflects the type and molecular status of their cell of origin, EV miRNA levels are altered under different biological and pathological conditions. In our previous study, we showed that TGFBR2 deficiency alters the proteome of MSI CRC-derived EVs (14). However, whether the expression status of TGFBR2 can modulate the repertoire of miRNAs in MSI tumor-derived EVs remains to be elucidated. To bridge this gap of knowledge, the present study aimed to decipher the TGFBR2-dependent miRNA profile of MSI tumor cells and their secreted EVs. An established MSI cell line model (HCT116-TGFBR2) was used (15), which enables the analysis of TGFBR2-dependent alterations in an isogenic background (Fig. 1). Small RNA sequencing (RNA-Seq) identified shared and distinct miRNA signatures in EVs and their parental MSI tumor cells that are regulated in a TGFBR2-dependent manner.

\section{Materials and methods}

Cell culture. The human epithelial HCT116 MSI CRC cell line was obtained from the European Collection of Cell Cultures (Salisbury, UK). The generation of the HCT116-TGFBR2 and HCT116-Tet-On cell lines has been described previously $(15,16)$. In both cell lines, the endogenous TGFBR 2 gene is inactivated (TGFBR2 deficient, dT) by homozygous frameshift mutations in a polyadenine tract (A10) within exon 3. HCT116-Tet-On cells are the parental cells of HCT116-TGFBR2. Both cell lines allow the constitutive expression of a doxycycline (dox)-responsive transactivator (16). HCT116-TGFBR2 cells confer dox-inducible expression of a TGFBR2 transgene (+dox, TGFBR2 proficient, pT), which was inserted as a single copy at a defined site in the HCT116-TGFBR2 genome (15). The HCT116-TGFBR2 and HCT116-Tet-On control cells were cultured in DMEM-F12 medium (Thermo Fisher Scientific, Inc., Waltham, MA, USA) supplemented with 10\% FBS
(Thermo Fisher Scientific, Inc.), $100 \mathrm{U} / \mathrm{ml}$ penicillin and $100 \mu \mathrm{g} / \mathrm{ml}$ streptomycin (Thermo Fisher Scientific, Inc.) in $5 \% \mathrm{CO}_{2}$ atmosphere at $37^{\circ} \mathrm{C}$. The Mycoplasma Detection kit (Minerva Biolabs, Berlin, Germany) was used, according to the manufacturer's protocol, to ensure that the cultured cells were free of mycoplasma contamination.

Extracellular vesicle isolation. Each of the four biological replicates was obtained from $5 \times$ T175 flasks containing $28 \times 10^{6}$ HCT116-TGFBR2 or HCT116-Tet-On cells. The cells were washed twice with PBS (Thermo Fisher Scientific, Inc.) and subsequently cultured in serum-free DMEM-F12 medium (17 ml/T175 flask). The HCT116-TGFBR2 cells were cultured in the presence of $0.5 \mu \mathrm{g} / \mathrm{ml}$ dox (+dox, pT; Sigma-Aldrich; Merck KGaA, Darmstadt, Germany) in order to induce the reconstituted expression of TGFBR2, or grown in the absence of dox (-dox, dT), which reflects the loss of TGFBR2 in MSI tumors. The ligand TGF- $\beta 1$ (10 ng/ml; Abcam, Cambridge, UK) was added to the dT and pT cells. In order to exclude any dox-related effects, two subsets of HCT116-Tet-On cells were also cultured in the presence or absence of dox. After $16 \mathrm{~h}$, the medium was collected from both conditions, and the cells were harvested and stored at $-80^{\circ} \mathrm{C}$. The medium was subjected to sequential centrifugation and precipitation as reported previously (14). Briefly, the supernatants were cleared by differential centrifugation $\left(480 \mathrm{x} \mathrm{g}, 5 \mathrm{~min}, 4^{\circ} \mathrm{C} ; 2,000 \mathrm{x} \mathrm{g}\right.$, $\left.10 \mathrm{~min}, 4^{\circ} \mathrm{C}\right)$, filtered $(0.22 \mu \mathrm{m})$, and concentrated (40-fold) using Vivaspin-20 tubes (Sartorius, Göttingen, Germany). The EVs were precipitated from this concentrate using Total Exosome Isolation Reagent (Thermo Fisher Scientific, Inc.) and pelleted by centrifugation $\left(20,000 \times \mathrm{g}, 1 \mathrm{~h}, 4^{\circ} \mathrm{C}\right)$. The EV pellets were lysed in QIAzol Lysis Reagent (Qiagen AB, Limburg, Netherlands) for RNA extraction, in RIPA buffer (50 mM Tris- $\mathrm{HCl} \mathrm{pH} 7.4,150 \mathrm{mM} \mathrm{NaCl}, 1 \%$ Triton $\mathrm{X}-100$, $1 \%$ Na-deoxycholate, $0.1 \%$ SDS, $0.1 \mathrm{mM} \mathrm{CaCl}_{2}$ and $0.01 \mathrm{mM}$ $\mathrm{MgCl}_{2}$ ) for protein extraction or resuspended in PBS for EV characterization.

Transmission electron microscopy (TEM). The EV suspensions (5 $\mu \mathrm{l})$ in PBS were left to settle onto 100 mesh formvar-coated copper grids (Plano, Wetzlar, Germany), contrasted with 2\% aqueous uranyl acetate (negative stain), air-dried and visualized using the JEM-1400 transmission microscope (JEOL, Ltd., Peabody, MA, USA) equipped with a Tietz 2k digital camera (TVIPS, Gauting, Germany) at $80 \mathrm{KV}$.

Nanoparticle tracking analysis. The size profiles and particle concentrations were assessed by nanoparticle tracking analysis (NTA) using the ZetaView PMX110 system with software 8.04.02 SP2 (Particle Metrix, Inning, Germany) according to the manufacturer's instructions. The EV suspensions were diluted 1:2,500 (v/v) in particle-free PBS and analyzed at 11 positions. Data acquisition thresholds were set to a shutter of 100 , a sensitivity of $95 \%$ and a frame rate of 30 frames $/ \mathrm{sec}$.

Protein extraction and Western blot analysis. The protein lysates of the EVs and cells were prepared in RIPA buffer supplemented with cOmplete Mini protease inhibitor cocktail inhibitor (Roche Diagnostics, Basel, Switzerland). Protein concentration was measured using the Bradford assay 
A

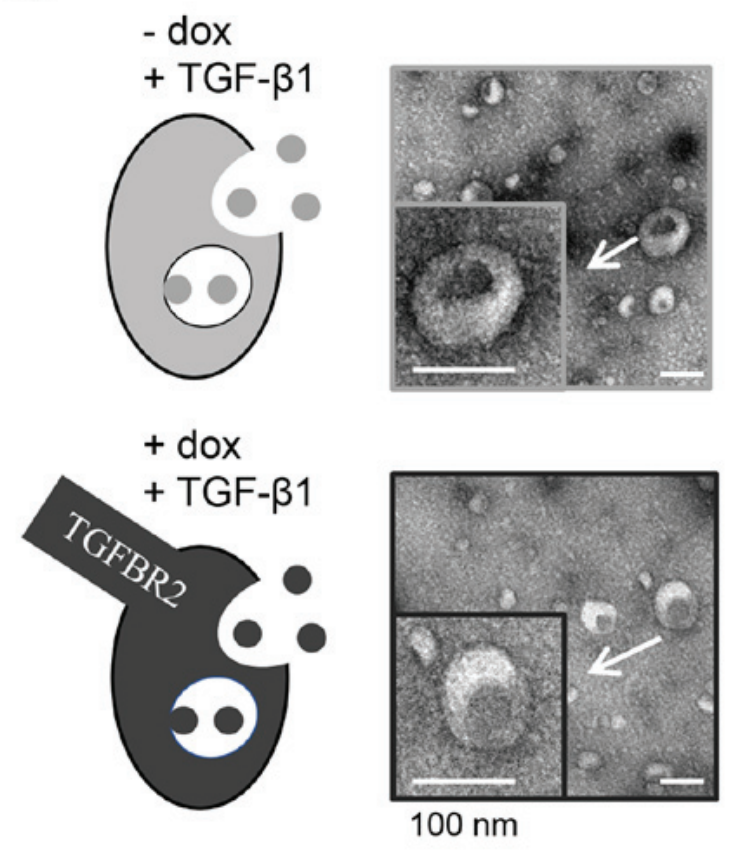

C

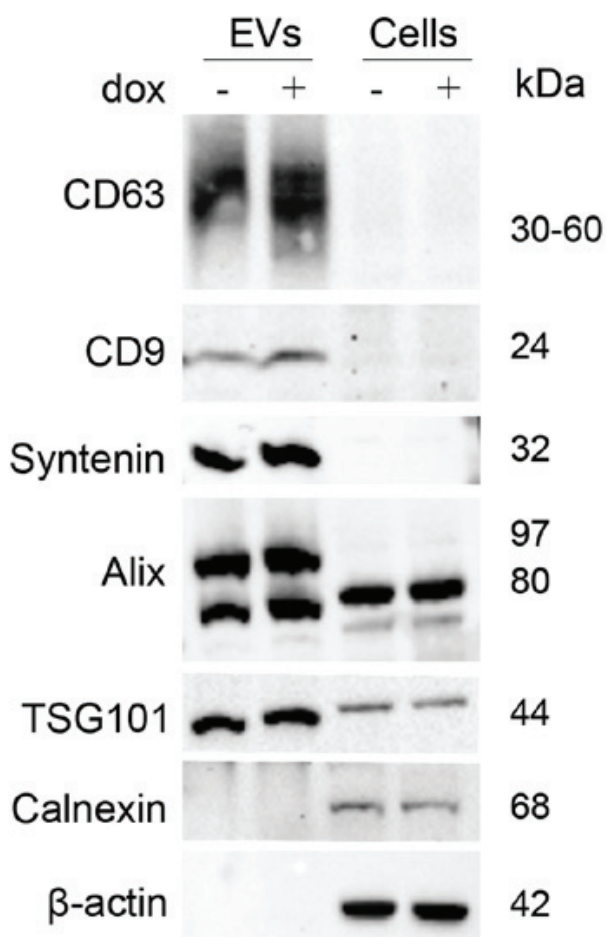

B

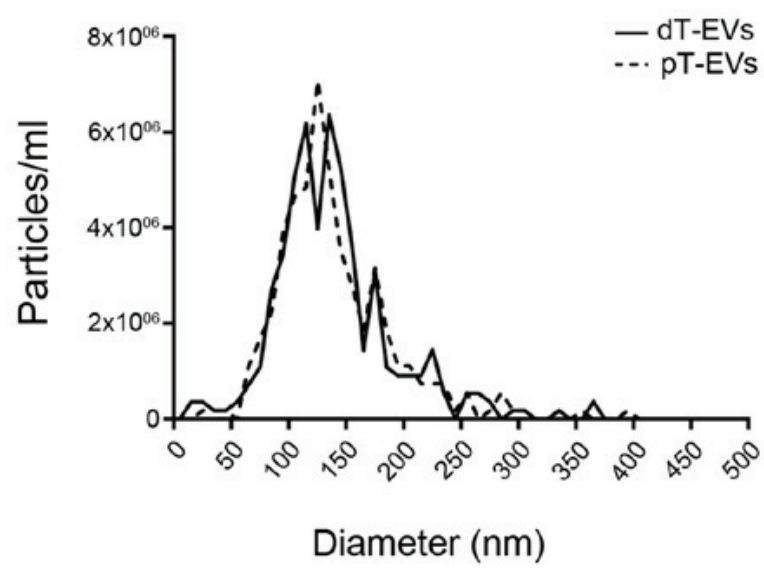

Figure 1. Schematic overview of the dox-inducible MSI model system and characterization of EVs. (A) HCT116-TGFBR2 cells lack TGFBR2 expression in the absence of dox (dT; light grey) and in the presence of TGF- $\beta 1$ ligand. Dox treatment leads to the reconstituted expression of TGFBR2 (pT; dark grey). EVs were isolated from dT and pT cells. TEM images show the size and shape of the isolated EVs (representative of six independent experiments). Insets indicate the magnification of representative vesicles marked by arrows. Scale bar=100 $\mathrm{nm}$. (B) Nanoparticle tracking analysis resulted in median sizes of $127.6 \mathrm{~nm}$ (pT EVs; dotted line) and $124.5 \mathrm{~nm}$ (dT EVs; black line) and comparable vesicle concentration (representative of four independent experiments). (C) Western blot analysis revealed EV marker expression in lysates of isolated EVs. Calnexin was used as a negative EV marker. $\beta$-actin served as a loading control for HCT116-TGFBR2 cell lysates. Protein sizes are indicated. MSI, microsatellite instability; TGFBR2, transforming growth factor- $\beta$ receptor type 2; EVs, extracellular vesicles; miR, microRNA; dox, doxycycline; dT, TGFBR2 deficient; pT, TGFBR2 proficient.

(Bio-Rad Laboratories, Inc., Hercules, CA, USA). Per sample, $50 \mu \mathrm{g}$ of protein was separated on $4-20 \%$ Bis-Tris gradient gels (Expedeon, Cambridge, UK) and blotted onto a nitrocellulose membrane (Thermo Fisher Scientific, Inc.). After blocking of the membranes in 5\% milk/TBST, the following primary antibodies were used: mouse anti-CD63 (1:500, clone MX-49.129.5, cat. no. sc-5276, Santa Cruz Biotechnology, Inc., Dallas, TX, USA), mouse anti-CD9 (1:200, clone C4, cat. no. sc-13118, Santa Cruz Biotechnology, Inc.), mouse anti- $\beta$-actin (1:2,000, clone C4, cat. no. 0869100-CF, MP Biomedicals, Santa Ana, CA, USA), rabbit anti-Alix (1:1,000, clone EPR15314-33, cat. no. ab186429, Abcam), mouse anti-TSG101 (1:500, clone 4A10, cat. no. ab83, Abcam), rabbit
anti-Syntenin (1:5,000, clone EPR8102, cat. no. ab133267, Abcam), goat anti-Calnexin (1:2,500, cat. no. WA-AF1179a, Biomol, Hamburg, Germany), mouse anti-TGFBR2 (1:300, clone D2, cat. no. sc-17799, Santa Cruz Biotechnology, Inc.), rabbit anti-Smad2 (1:1,000, clone 86F7, cat. no. 3122S, Cell Signaling Technology, Inc., Danvers, MA, USA), and rabbit anti-phosphorylated (p)Smad2 (1:1,000, Ser465/467, cat. no. 3101, Cell Signaling Technology, Inc.). The primary antibodies were diluted in 5\% milk/TBST and incubated with membranes overnight at $4^{\circ} \mathrm{C}$. The blots were then washed with TBST and incubated with a sheep anti-mouse-IgG HRP (1:5,000, cat. no. NXA931, GE Healthcare, Chicago, IL, USA), goat anti-rabbit-IgG HRP (1:2,500, cat. no. 7074, Promega 
Corporation, Madison, WI, USA) or donkey anti-goat-IgG HRP (1:1,000, cat. no. sc-2020, Santa Cruz Biotechnology, Inc.) secondary antibody for $1 \mathrm{~h}$ at room temperature. The signals were detected using Western Lightning Plus ECL (Perkin Elmer, Inc., Waltham, MA, USA) and a ChemiDoc MP system (Bio-Rad Laboratories, Inc.).

RNA extraction and quality control. Total RNA was extracted from the EVs and parental cells using the miRNeasy Mini kit (Qiagen $\mathrm{GmbH})$. The cells and EVs were homogenized in $700 \mu \mathrm{l}$ QIAzol Lysis Reagent (Qiagen $\mathrm{GmbH}$ ) and total RNA was isolated according to the manufacturer's instructions. RNA was eluted in $30 \mu \mathrm{l}$ RNase-free water. The yield and size distribution of the isolated RNAs were assessed by capillary electrophoresis using the RNA Nano and RNA 6000 Pico kit on the 2100 Bioanalyzer (Agilent Technologies GmbH, Waldbronn, Germany). Integrity values for cellular RNAs were calculated using the Bioanalyzer's RNA integrity number (RIN) algorithm. The RIN algorithm is designed to estimate RNA quality for eukaryotic cells but cannot be used for quality assurance of EV RNA due to lack of full-length $28 \mathrm{~S}$ and $18 \mathrm{~S}$ rRNA. For reverse transcription-quantitative polymerase chain reaction (RT-qPCR) analysis, the RNA concentrations were determined using a NanoDrop instrument (Thermo Fisher Scientific, Inc.).

Library preparation and small RNA sequencing. The total RNA eluates $(30 \mu \mathrm{l})$ were vacuum-evaporated and resuspended in $8 \mu \mathrm{l}$ nuclease-free water. For small RNA library preparation, $6 \mu 1$ of total RNA and the NEBNextMultiplex Small RNA Library Prep Set for Illumina (New England BioLabs, Ipswich, MA, USA) were utilized according to the manufacturer's instructions, including recommended adjustments for low RNA input. The PCR products were purified using the MinElute PCR Purification kit (Qiagen $\mathrm{GmbH}$ ) prior to library evaluation using the DNA $1000 \mathrm{kit}$ (Agilent Technologies GmbH) and the Bioanalyzer 2100. To allow size selection of miRNA-containing fragments at a length of 130-150 base pairs, the pooled library was separated on a high-resolution 4\% agarose gel (MetaPhor Agarose, Lonza Rockland, Rockland, ME, USA) at $150 \mathrm{~V}$ at $4^{\circ} \mathrm{C}$, and the corresponding bands were cut from the gel. Following gel extraction with the MinElute Gel Extraction kit (Qiagen $\mathrm{GmbH}$ ), capillary gel electrophoresis was performed using the Bioanalyzer DNA High Sensitivity kit (Agilent Technologies $\mathrm{GmbH}$ ) to control for the size and purity of the final library. Small RNA sequencing was conducted on a HiSeq 2500 using the HiSeq Rapid SBS Kit v2 (Illumina, Inc., San Diego, CA, USA) and 50 cycles of single-end sequencing-by-synthesis.

Data analysis. Raw data was imported into FastQC software (Babraham Bioinformatics, Cambridge, UK, version 0.11.7) to evaluate technical sequencing parameters including the per base sequence quality, indicated by the Phred quality score, and sequence length distribution (17). Adaptor sequences added to the 3'-end during library preparation were trimmed using Btrim (18). Reads with a length of $<16$ nucleotides ('Short') were excluded to avoid false positive mappings. Reads corresponding to other RNA classes [ribosomal ('rRNA'), small nuclear ('snRNA'), small nucleolar (snoRNA)' and transfer ('tRNA')] were identified by mapping to sequences obtained from RNAcentral, v9 (19), and omitted in subsequent analyses. The filtered reads were then aligned to human miRNA precursor sequences obtained from miRBase, release 22 (20), allowing for one mismatch using Bowtie (21). The unmapped reads were classified as 'Unmapped' and disregarded. The read count table generated by the sum of the hits per miRNA sequence was loaded into $\mathrm{R}$ (version 3.5.1; https://www.r-project.org/) and used as input for the Bioconductor package DESeq2 (version 1.20.0; https://bioconductor.org/packages/release/bioc/html/DESeq2. html) (22). Differential gene expression (DGE) analysis was conducted to evaluate the regulation of gene expression in a TGFBR2-dependent manner. Fold changes (dT EVs/pT EVs and dT cells/pT cells) were log2-transformed for statistical data analysis. The obtained P-values were adjusted for multiple comparisons according to Benjamini and Hochberg (23). The baseMean of each miRNA, which reflects the mean expression across all samples, was set to $\geq 20$ reads. An adjusted P-value $(p$-adj) of $P \leq 0.05$ and a $\log 2$ fold change $(F C) \geq|0.585|$ were applied as cut-off values. For further calculation and visualization of the DESeq 2 results, the following packages were used: data.table, RColorBrewer, gplots, ggplot2, genefilter, ggfortify, VennDiagram, dendextend, reshape2, and scales. Outliers were detected using Cook's distance test (22). Exploratory data analysis was performed by displaying the regularized logarithm-transformed data in principal component analysis (PCA), heatmap analysis, and hierarchical clustering by Euclidean distances. Interaction network analysis was performed using miRNet (https://www.mirnet.ca) (24) based on conventional hypergeometric tests and the Reactome pathway database (https://reactome.org) (25).

$R T$-qPCR analysis. Total RNA (100 ng) was subjected to RT reactions using the miRCURY LNA RT kit (Qiagen $\mathrm{GmbH}$ ) according to the manufacturer's protocol. UniSp6 was used as a spike-in control. For real-time PCR analysis, the cDNA products were diluted 1:10 (v/v) in nuclease-free water. qPCR was performed in a total volume of $10 \mu \mathrm{l}$ using the miRCURY LNA SYBR Green PCR kit (Qiagen $\mathrm{GmbH}$ ). The following miRCURY LNA miRNA PCR Assay primers (Qiagen $\mathrm{GmbH}$ ) were used: hsa-miR-376a-3p (cat. no. YP00204508), hsa-miR-381-3p (cat. no. YP00205887), hsa-miR-379-5p (cat. no. YP00205658), hsa-miR-181a-2-3p (cat. no. YP00204142), hsa-miR-30d-5p (cat. no. YP00206047), hsa-miR-362-3p (cat. no. YP00205612), hsa-miR-92b-3p (cat. no. YP00204384), hsa-miR-25-3p (cat. no. YP00204361) and hsa-miR-744-5p (cat. no. YP00204663). Nuclease-free water was used as a no template negative control. Each PCR was performed in triplicate on a StepOnePlus Real-Time PCR system (Thermo Fisher Scientific, Inc.) at $95^{\circ} \mathrm{C}$ for $2 \mathrm{~min}$, followed by 40 cycles of $95^{\circ} \mathrm{C}$ for $10 \mathrm{sec}$ and $56^{\circ} \mathrm{C}$ for $1 \mathrm{~min}$. The specificity of amplification was assessed by melting curve analysis. Raw quantification cycle $(\mathrm{Cq})$ values were generated and data were analyzed using StepOnePlus software (version 2.1; Thermo Fisher Scientific, Inc.). The baseline was set automatically and the threshold values were adjusted manually. Relative quantification was conducted using the $2^{-\Delta \Delta \mathrm{Cq}}$ method $(26,27)$. Reference candidates stably expressed across all replicates of EVs and parental MSI cells 
were identified from next generation sequencing data using NormFinder (https://moma.dk/normfinder-software) (28) and GeNorm (https://genorm.cmgg.be) (29). miR-30d-5p, miR-362-3p, miR-744-5p, and miR-92b-3p and miR-25-3p were used as endogenous controls for normalizing target miRNA expression levels in EVs and MSI cells, respectively. Five significantly regulated miRNAs were selected for validation (cells: miR-381-3p, miR-379-5p and miR-181a-2-3p; EVs: miR-381-3p and miR-376a-3p). Statistical significance $(\mathrm{P} \leq 0.05)$ was assessed using Student's t-test.

Statistical analysis. Statistical calculations were performed in SigmaPlot (version 11.0; Systat Software, San Jose, CA, USA), R software (version 3.5.1) and Prism (version 7.00; GraphPad Software, Inc., La Jolla, CA, USA). The quantitative miRNA expression levels identified by RNA-Seq were compared between the dT and pT samples using the Bioconductor Package DESeq2 (version 1.20.0). Fold changes (dT EVs/pT EVs and $\mathrm{dT}$ cells/pT cells) were $\log 2$-transformed for statistical data analysis and the obtained $\mathrm{P}$-values were adjusted for multiple comparisons according to Benjamini and Hochberg (23). A p-adj of $\mathrm{P} \leq 0.05$ and a $\log 2 \mathrm{FC} \geq|0.585|$ were applied as cut-off values. For the qPCR data, statistical significance between the quantitative miRNA expression levels was assessed using an unpaired, two-tailed Student's t-test. $\mathrm{P} \leq 0.05$ was considered to indicate a statistically significant difference. Data in the text and in tables are presented as the mean \pm standard deviation.

\section{Results}

General extracellular vesicle characteristics are not altered by the cellular expression of TGFBR2. In order to investigate TGFBR2-dependent alterations in MSI tumor cells and their secreted EVs, the well-characterized HCT116-TGFBR2 cell line was used as a model system (15). In the absence of dox, HCT116-TGFBR2 cells are TGFBR2 deficient (-dox, dT), which reflects the condition of most primary MSI tumors that have lost receptor expression during tumor progression. By contrast, in the presence of dox, these cells show reconstituted receptor expression (+dox, pT), which allows the identification of TGFBR2-dependent alterations in an isogenic background. Prior to EV preparation, reconstitution of a functional TGF- $\beta$ pathway was examined by Western blot analysis (Fig. S1). In the absence of dox and in the presence of the TGF- $\beta 1$ ligand, the HCT116-TGFBR2 cells lacked TGFBR2 expression and pSmad 2 was almost undetectable. However, in the presence of dox and the ligand, these cells showed reconstituted expression of TGFBR2 and downstream signaling, as demonstrated by the activation of $\mathrm{pSmad} 2$. Following establishment of the induction control, the EVs were isolated from the pT cells and dT cells, and their morphology, size, concentration and EV marker protein expression were assessed by different methods (Fig. 1) in compliance with the MISEV2018 recommendations (30). First, using TEM, single EVs appeared as round, cup-shaped, lipid bilayer-enclosed structures within a size range of $30-150 \mathrm{~nm}$ in all preparations (Fig. 1A). Second, NTA analysis independently confirmed the observed size distribution of $127.6 \mathrm{~nm}$ (mean $139.5 \pm 52.3 \mathrm{~nm}$ ) for dT EVs and $124.5 \mathrm{~nm}$ (mean $140.6 \pm 52.4 \mathrm{~nm}$ ) for pT EVs (Fig. 1B). The initial particle concentrations were calculated, resulting in $4.6 \times 10^{4}$ (dT EVs) and $4.5 \times 10^{4}$ (pT EVs) particles per ml cell culture supernatant. Third, Western blot analysis revealed the expression of EV-specific and cell-specific marker proteins (Fig. 1C). Tetraspanins CD63 and CD9 were expressed in lysates of the EVs but not in lysates of the parental cells. The EV marker protein Syntenin was also found to be exclusively detected in EV lysates. TSG101 and Alix were enriched in the protein lysates of EVs compared with those of their parental cells. The endoplasmic reticulum protein Calnexin and cytoskeletal $\beta$-actin served as controls. Both were detected in the cell lysates but not in the EV lysates, excluding any contamination of EV lysates with cellular debris. These results demonstrate the successful enrichment of a comprehensively characterized population of EVs from dT cells and pT cells, and showed the reproducibility of EV isolation, thus enabling subsequent analysis of miRNA profiles in a TGFBR2-dependent manner.

Technical assessment reveals high RNA quality and sequencing accuracy. Subsequently, total RNA was isolated from four biological replicates of dT cells and pT cells and from the dT EVs and pT EVs derived thereof. For comparative RNA-Seq analysis, high quality RNA of sufficient quantity is of utmost importance. When total cellular RNA was analyzed by capillary electrophoresis, similar levels of RNA were detected in pT cells $(1.9 \pm 1.0 \mu \mathrm{g})$ and dT cells $(2.3 \pm 1.2 \mu \mathrm{g})$. Subsequent examination of the EVs shed by these cells revealed lower and marginally different yields of RNA from $\mathrm{pT}$ EVs $(344.8 \pm 129.6 \mathrm{ng})$ and dT EVs $(254.4 \pm 107.2 \mathrm{ng})$ (Table I). Further control of RNA integrity resulted in a mean RINs of $9.98 \pm 0.05$ (pT cells) and 9.85 \pm 0.13 (dT cells), indicating an RNA quality of high grade (Table I). Small RNA was then profiled by RNA-Seq. Based on this analysis, all samples had a Phred score $\geq 38$ (mean 39.04), reflecting a high sequencing accuracy (31). The length distribution of the sequencing data exposed a mono-peak at 22 nucleotides in all groups (Fig. S2). Libraries of $5.56 \times 10^{6} \pm 6.40 \times 10^{5}$ (pT cells), $5.47 \times 10^{6} \pm 6.87 \times 10^{5}$ (dT cells), $6.95 \times 10^{6} \pm 1.28 \times 10^{6}$ (pT EVs) and $6.10 \times 10^{6} \pm 5.11 \times 10^{5}$ (dT EVs) reads were generated (Table I). Although sequencing of RNA from EVs resulted in higher read counts compared with that of cellular RNA, the TGFBR2 expression status did not significantly alter the library size (Table I; Fig. S2).

Subsequently, the reads were mapped to distinct classes of small non-coding RNAs (Fig. 2). Although the cells showed a higher amount of snoRNA, the mapping distribution indicated an enrichment of tRNA and snRNA in the EVs. Reads mapping to miRNAs accounted for the major proportion (60.6-63.9\%) of all reads in all four groups (Fig. 2, Table I). In particular, $3.5 \times 10^{6} \pm 3.6 \times 10^{5}$ (pT cells), $3.5 \times 10^{6} \pm 4.8 \times 10^{5}$ (dT cells), $4.2 \times 10^{6} \pm 8.4 \times 10^{5}$ (pT EVs) and $3.8 \times 10^{6} \pm 4.8 \times 10^{5}$ (dT EVs) reads mapped to miRNAs (Table I). Following application of an expression threshold of at least 20 reads per replicate for each miRNA, similar numbers of individual miRNAs were identified in all four groups (Table I). Taken together, these analyses indicated a high RNA quality and sequencing accuracy, allowing further investigation of TGFBR2-dependent miRNA profiles in EVs and parental MSI tumor cells.

Clustering of miRNA profiles is determined by the expression of TGFBR2. A total of 471 distinct miRNAs were identified among all four groups at a qualitative expression level. Venn 
Table I. Technical assessment of small RNA sequencing.

Parental MSI cells

\begin{tabular}{|c|c|c|c|c|}
\hline \multirow[b]{2}{*}{ Technical parameter } & & \\
\hline & pT cells & dT cells & pTEVs & dT EVs \\
\hline $\mathrm{RNA}(\mathrm{ng}) \pm \mathrm{SD}$ & $1,914.4 \pm 1,000.6$ & $2,304.8 \pm 1,202.4$ & $344.8 \pm 129.6$ & $254.4 \pm 107.2$ \\
\hline $\mathrm{RIN} \pm \mathrm{SD}$ & $9.98 \pm 0.05$ & $9.85 \pm 0.13$ & NA & NA \\
\hline Libraries (reads) $\pm \mathrm{SD}$ & $5.56 \times 10^{6} \pm 6.40 \times 10^{5}$ & $5.47 \times 10^{6} \pm 6.87 \times 10^{5}$ & $6.95 \times 10^{6} \pm 1.28 \times 10^{6}$ & $6.10 \times 10^{6} \pm 5.11 \times 10^{5}$ \\
\hline miRNA reads $(\%)$ & 63 & 64 & 61 & 63 \\
\hline $\begin{array}{l}\text { Total miRNAs } \\
\text { (reads) } \pm \mathrm{SD}\end{array}$ & $3.5 \times 10^{6} \pm 3.6 \times 10^{5}$ & $3.5 \times 10^{6} \pm 4.8 \times 10^{5}$ & $4.2 \times 10^{6} \pm 8.4 \times 10^{5}$ & $3.8 \times 10^{6} \pm 4.8 \times 10^{5}$ \\
\hline Numbers of miRNAs & 360 & 357 & 380 & 367 \\
\hline
\end{tabular}

Presented numbers are mean values calculated from four biological replicates. TGFBR2, transforming growth factor- $\beta$ receptor type 2; dT, TGFBR2 deficient; pT, TGFBR2 proficient; EVs, extracellular vesicles; RIN, RNA integrity number; miRNA, microRNA; NA, not applicable.

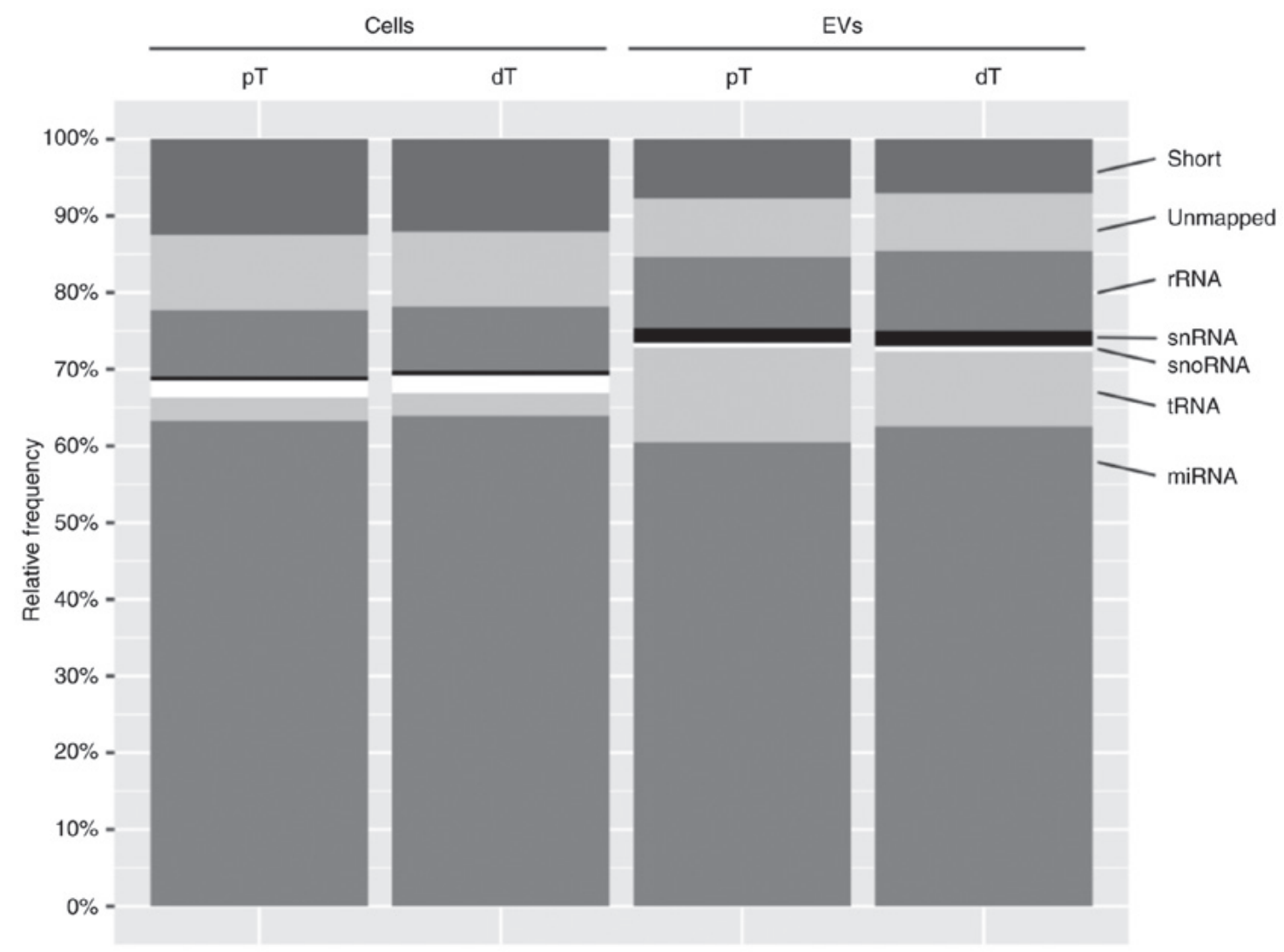

Figure 2. Mapping statistics for different classes of small non-coding RNA. Data are expressed as the mean mapping percentages (relative frequency) calculated from four biological replicates of pT cells and EVs and dT cells and EVs. Short, sequence <16 nt long; unmapped, sequence did not align to human small RNA; rRNA, ribosomal RNA; snRNA, small nuclear RNA; snoRNA, small nucleolar RNA; tRNA, transfer RNA; miRNA microRNA; dT, TGFBR2 deficient; pT, TGFBR2 proficient; TGFBR2, transforming growth factor- $\beta$ receptor type 2 ; EVs, extracellular vesicles.

diagram comparison showed the presence of individual and shared miRNA subsets among these groups (Fig. 3). The majority $(263 / 471 ; 56 \%)$ of the identified miRNAs were shared between the EVs and parental MSI tumor cells.

In addition to this overlap, 100 miRNAs were exclusively detected in the EVs, of which 82 candidates were identified in both dT EVs and pT EVs. Several miRNAs were exclusively expressed in the dT EVs $(n=4$; miR-122-5p, miR-92a-1-5p, miR-1343-3p and miR-146b-3p) or pT EVs (n=14; miR-20b-5p, miR-616-5p, miR-19b-1-5p, miR-561-3p, miR-539-3p, miR-382-3p, miR-6516-3p, miR-3136-5p, miR-96-3p, miR-3140-3p, miR-1249-3p, miR-106a-5p, miR-548ai/-570-5p and miR-3065-5p).

By contrast, 81 miRNAs were exclusively detected in the MSI tumor cells, of which 71 miRNAs were detected in both $\mathrm{dT}$ and $\mathrm{pT}$ cells. TGFBR2-dependent unique expression clusters were also identified in the dT cells $(n=4 ; \mathrm{miR}-4429$, miR-2355-3p, miR-320e and miR-371a-5p) and pT cells $(n=6$; 


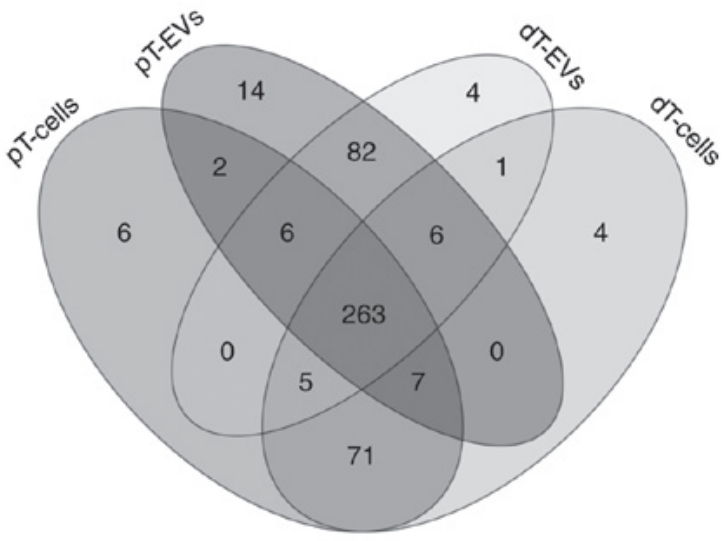

Figure 3. Qualitative assessment of microRNA expression. Numbers refer to identified miRNAs either unique to a group or part of an intersection in the Venn diagram comparison. TGFBR2, transforming growth factor- $\beta$ receptor type 2; dT, TGFBR2 deficient; pT, TGFBR2 proficient; EVs, extracellular vesicles.

miR-505-5p, miR-411-5p, miR-223-3p, miR-5001-5p, miR-184 and $\mathrm{miR}-495-3 \mathrm{p})$.

One candidate, miR-200c-5p, was identified in both the dT EVs and dT cells but was not detected in samples derived from the TGFBR2-proficient condition. By contrast, two candidates (miR-323a-3p and miR-382-5p) were only identified in the pT EVs and parental pT cells.

Based on these results, exploratory data analysis was performed to assess the clustering of individual samples. Hierarchical cluster analysis (HCA) and principal component analysis (PCA) identified one of the four biological replicates in the EV group as an outlier. The altered miRNA expression pattern of this outlier (EV_V1) was also indicated by Cook's distance test (22) and was thus excluded from further analysis. As shown in Fig. 4A, PCA clearly separated all biological replicates of the EVs from the parental MSI cells along the first principal component (PC1) with a variance of $81.04 \%$. This clustering indicated substantial differences in the expression of miRNAs between the EVs and parental cells. The second principle component (PC2) accounted for $2.68 \%$ of the overall variance. The distances of miRNA expression were higher between the EVs and parental cells than between TGFBR 2 conditions within one sample category. In addition to PCA, miRNA expression was analyzed by HCA (Fig. 4B). The HCA dendrogram showed a distinct fractionation of cells and EVs in addition to visible cluster distinction depending on the TGFBR2 expression status. The heatmap illustrated clear cluster changes in miRNAs between EVs and their parental cells (Fig. 4B). Considering the grouping performance of PCA and HCA, the miRNA profiles of the EVs and the cells differed in a TGFBR2-dependent manner.

TGFBR2 deficiency modulates miRNA profiles in EVs and MSI tumor cells. In the subsequent step, differential gene expression analyses were performed to quantify miRNA candidates with significant TGFBR2-mediated expression differences. Sequencing data were subjected to DESeq2 analysis in order to compare miRNA expression between the $\mathrm{dT}$ and $\mathrm{pT}$ conditions in EVs and parental cells separately. Fold changes (dT/pT) were calculated to quantify the differential expression patterns. Applying stringent filtering criteria
( $\log 2 \mathrm{FC} \geq 10.585 \mid$, adj. $\mathrm{P} \leq 0.05$, mean expression $\geq 20$ reads), DESeq2 analysis revealed TGFBR2-regulated miRNAs in HCT116-TGFBR2 cells and their EVs (Fig. 5).

In the EVs, the expression of 10 miRNAs was regulated in a TGFBR2-dependent manner. Of the candidates, 7/10 (miR-376a-3p, miR-381-3p, miR-410-3p, miR-376c, miR-889-3p, miR-323a-3p and miR-376b-3p) were downregulated in the dT EVs, the remaining 3/10 miRNAs (miR-320d, miR-320c and miR-3187-3p) were upregulated in the dT EVs, as indicated by positive $\log 2 \mathrm{FC}$ (Fig. 5A, Table II). In silico network analysis predicted interactions between these 10 TGFBR2-regulated miRNAs and 1,022 target genes (Fig. S3) (24). Subsequent functional enrichment analysis revealed that signaling by the TGF- $\beta$ receptor complex was the most affected $(\mathrm{P}=0.0002)$ pathway (Table SI). More specifically, among all identified target genes, 18 candidates appeared to mediate signal transduction downstream of TGFBR2.

When miRNA abundance in parental MSI cells was examined, 15 miRNAs were regulated in a TGFBR2-dependent manner (Fig. 5B). In particular, 2/15 miRNAs (miR-31-3p and miR-4521) were upregulated and 13/15 miRNAs (miR-381-3p, miR-409-3p, miR-379-5p, miR-543, miR-181a-2-3p, miR-411-5p, miR-382-5p, miR-654-3p, miR-134-5p, miR-323a-3p, miR-495-3p, miR-889-3p and miR-372-3p) were downregulated in dT cells (Table II). Apart from these differences, three TGFBR2-dependent miRNAs (miR-381-3p, miR-323a-3p and miR-889-3p) appeared to be downregulated in both dT EVs (3/10) and dT cells (3/15) (Fig. 5).

Taken together, the tumor driver mutation affecting TGFBR2 impacts the expression of 25 miRNAs ( $E V \mathrm{n}=10$; cells $n=15$ ). From these 25 candidates, three candidates were downregulated in both dT cells and their dT EVs, whereas the majority of TGFBR2-dependent miRNAs (19/25) were specifically regulated in MSI cells and their secreted EVs.

Differential miRNA expression is mediated by reconstituted expression of TGFBR2 rather than dox treatment. To validate the TGFBR2-regulated miRNAs in the MSI-derived EVs, the expression levels of miR-381-3p and miR-376a-3p were examined by RT-qPCR analysis (Fig. 6A, Table III). With a FC of 0.55 , miR-381-3p exhibited significantly $(\mathrm{P}=0.006)$ lower expression in the dT EVs. Similarly, miR-376a-3p exhibited significantly (FC 0.59; $\mathrm{P}=0.013$ ) lower expression levels in the dT EV samples compared with those in the pT EV samples, thereby confirming the results of the small RNA-Seq analysis.

For validation of the TGFBR2-regulated miRNAs in the parental MSI HCT116-TGFBR2 cells (Fig. 6B, Table III), the expression levels of miR-381-3p, miR-379-5p and miR-181-2-3p were investigated. A statistically significant (FC 0.39; $\mathrm{P}=0.005$ ) downregulation of miR-381-3p was found in the dT cells compared with that in the pT cells. For the two cellular candidates, miR-379-5p (FC 0.39; $\mathrm{P}=0.154)$ and miR-181a-2-3p (FC 0.80; $\mathrm{P}=0.745)$, a trend towards lower expression in dT cells was observed, although this was not statistically significant (Fig. 6B).

In order to investigate potential changes of miRNA profiles caused by dox treatment, EVs were isolated from the +doxand -dox-treated HCT116-Tet-On control cells. Differential miRNA expression was not observed in these control cells 

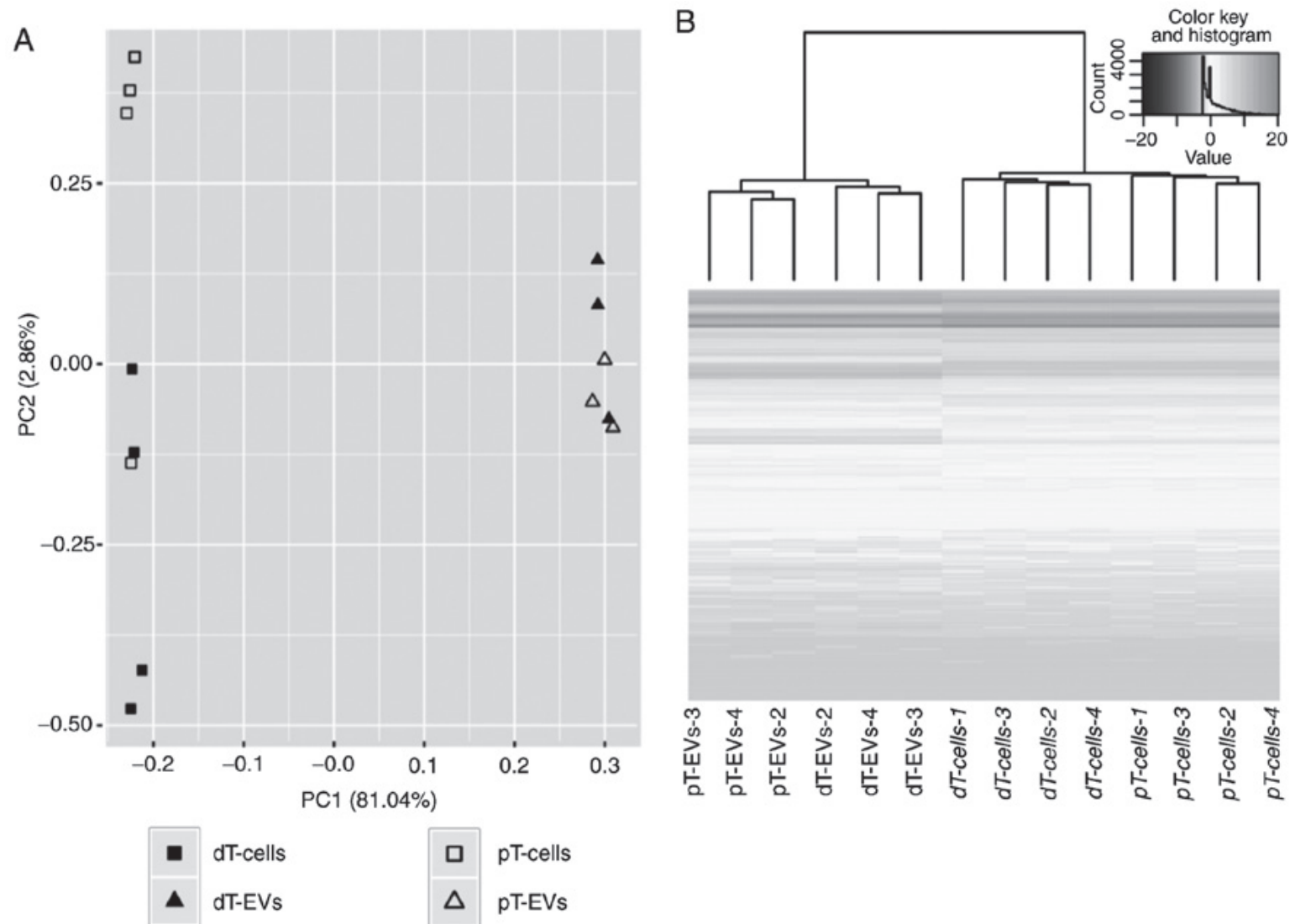

Figure 4. Exploratory data analysis of cellular and EV microRNAs based on TGFBR2 expression status. (A) PCA demonstrated a clear separation of all biological replicates from HCT116-TGFBR2 cells $(n=4)$ and their EVs $(n=3)$ along PC1 with a variance of $81.04 \%$. The PC2 accounted for $2.68 \%$ of overall variance. (B) Hierarchical cluster analysis revealed a clear fractionation of replicates (indicated by numbers) from MSI tumor cells and their EVs with accurate cluster distinction depending on the expression of TGFBR2. TGFBR2, transforming growth factor- $\beta$ receptor type 2 ; dT, TGFBR2 deficient; pT, TGFBR2 proficient; PC, principal component. EVs, extracellular vesicles.
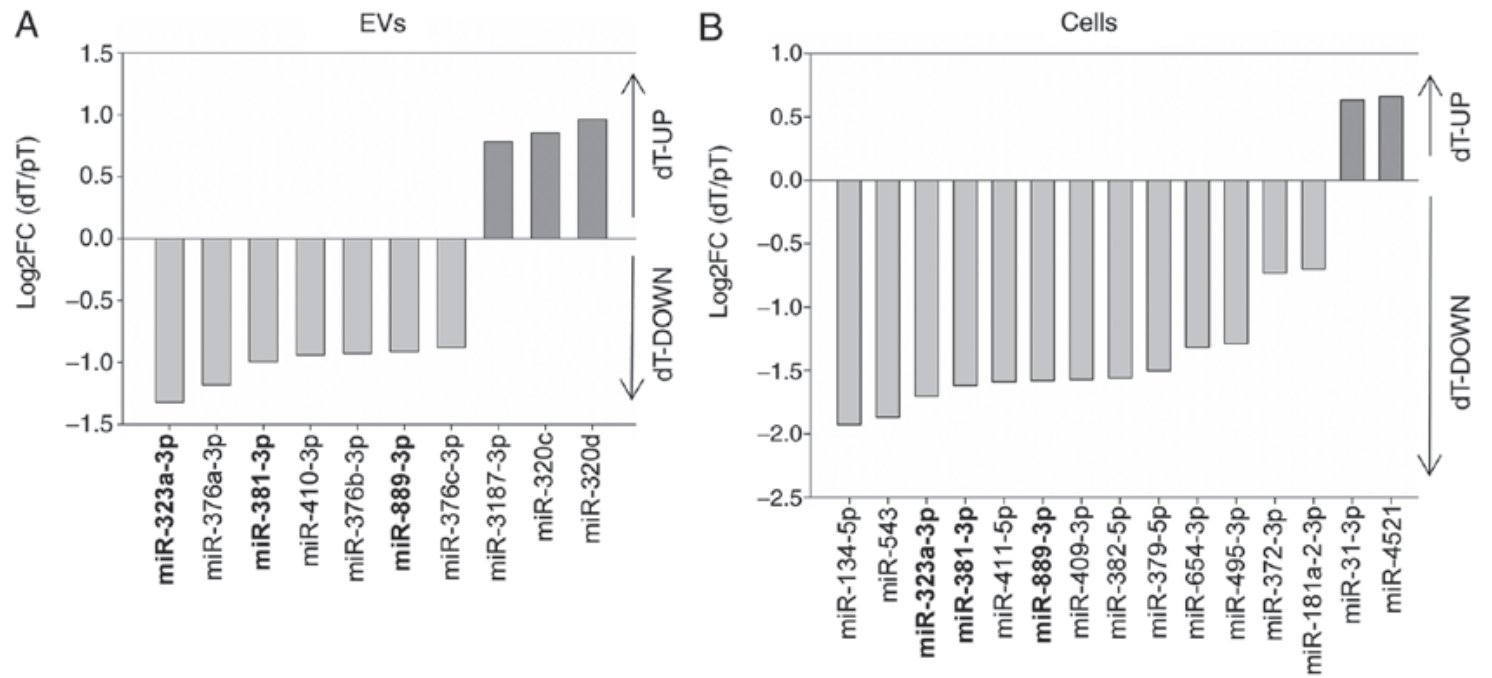

Figure 5. Quantitative differences in miRNA expression caused by TGFBR2 deficiency. Vertical bars indicate the $\log 2 \mathrm{FC}(\mathrm{dT} / \mathrm{pT})$ of regulated miRNAs in (A) EVs ( $\mathrm{n}=10)$ and (B) parental cells $(\mathrm{n}=15)$. Negative $\log 2 \mathrm{FC}$ values (light grey) indicate downregulation (dT-DOWN), whereas positive log2FC values (dark grey) indicate upregulation (dT-UP) upon TGFBR2 deficiency. TGFBR2-regulated miRNAs shared between EVs and cells (bold). miRNA/miR, microRNA; TGFBR2, transforming growth factor- $\beta$ receptor type 2; EVs, extracellular vesicles; dT, TGFBR2 deficient; pT, TGFBR2 proficient; FC, fold change.

or their derived control EVs (Table III), thus excluding any dox-related effect on the identified and successfully validated TGFBR2-dependent miRNA expression signatures in the EVs and parental MSI tumor cells.

\section{Discussion}

The majority of cases of MSI CRC are affected by the mutational inactivation of TGFBR2, allowing MSI tumor cells 
Table II. TGFBR2-regulated candidates in EVs and their parental MSI tumor cells.

\begin{tabular}{|c|c|c|c|}
\hline miRNA & $\log 2 \mathrm{FC}$ & p-adj & Mean expression \\
\hline \multicolumn{4}{|l|}{ EVs $(n=10)$} \\
\hline $\operatorname{miR}-323 a-3 p$ & -1.32 & 0.0100 & 39.80 \\
\hline $\operatorname{miR}-376 a-3 p$ & -1.18 & $2.46 \times 10^{-5}$ & 971.86 \\
\hline miR-381-3p & -0.99 & 0.0004 & 810.97 \\
\hline $\operatorname{miR}-410-3 p$ & -0.94 & 0.0039 & 108.53 \\
\hline $\operatorname{miR}-376 b-3 p$ & -0.93 & 0.0277 & 82.14 \\
\hline miR-889-3p & -0.91 & 0.0071 & 115.66 \\
\hline $\operatorname{miR}-376 c-3 p$ & -0.88 & 0.0065 & 145.53 \\
\hline $\operatorname{miR}-3187-3 p$ & 0.78 & 0.0253 & 142.28 \\
\hline $\operatorname{miR}-320 \mathrm{c}$ & 0.85 & 0.0100 & $2,216.98$ \\
\hline $\operatorname{miR}-320 \mathrm{~d}$ & 0.96 & 0.0059 & $3,474.59$ \\
\hline \multicolumn{4}{|c|}{ Parental MSI cells $(n=15)$} \\
\hline miR-134-5p & -1.93 & $5.81 \times 10^{-5}$ & 20.39 \\
\hline miR-543 & -1.872 & $2.54 \times 10^{-17}$ & 75.29 \\
\hline miR-323a-3p & -1.70 & 0.0005 & 20.47 \\
\hline miR-381-3p & -1.62 & $1.23 \times 10^{-53}$ & 548.64 \\
\hline $\operatorname{miR}-411-5 p$ & -1.59 & $8.95 \times 10^{-6}$ & 33.38 \\
\hline miR-889-3p & -1.58 & 0.0037 & 20.05 \\
\hline miR-409-3p & -1.575 & $5.33 \times 10^{-22}$ & 237.54 \\
\hline miR-382-5p & -1.56 & $3.55 \times 10^{-5}$ & 32.84 \\
\hline $\operatorname{miR}-379-5 p$ & -1.50 & $1.43 \times 10^{-18}$ & 149.75 \\
\hline $\operatorname{miR}-654-3 p$ & -1.32 & $4.56 \times 10^{-5}$ & 37.48 \\
\hline miR-495-3p & -1.29 & 0.0024 & 35.67 \\
\hline miR-372-3p & -0.73 & 0.0299 & 52.31 \\
\hline miR-181a-2-3p & -0.70 & $8.21 \times 10^{-12}$ & 506.83 \\
\hline $\operatorname{miR}-31-3 p$ & 0.63 & 0.0019 & 140.31 \\
\hline miR-4521 & 0.66 & 0.0192 & 109.83 \\
\hline
\end{tabular}

Mean expression of $\geq 20$ reads/miRNA, adj. $\mathrm{P} \leq 0.05, \log 2 \mathrm{FC} \geq|0.585|$ were applied as cut-off values. Data in each column $(\log 2 \mathrm{FC}, \mathrm{p}$-adj and mean expression) represent the mean values calculated from three (EVs) or four (cells) biological replicates. All values were determined using the DESeq2 algorithm. Negative $\log 2 \mathrm{FC}$ values indicate downregulation by loss of TGFBR2, whereas positive $\log 2 \mathrm{FC}$ values indicate upregulation by lack of TGFBR2. miR, microRNA; TGFBR2, transforming growth factor- $\beta$ receptor type 2 ; EVs, extracellular vesicles; MSI, microsatellite instability; $\mathrm{p}$-adj, adjusted P-value; FC, fold change.

to evade normal TGF- $\beta$ signaling and gain oncogenic growth advantages. In an attempt to dissect the inherent complexity of molecular and biological mechanisms associated with TGFBR2 signaling in normal and tumor cells, the present study established a genetically modified MSI CRC cell line, referred to as HCT116-TGFBR2. This MSI model cell line allows inducible, reconstituted expression of a wild-type TGFBR2 transgene and controlled activation of canonical TGF- $\beta$ signal transduction in an isogenic background (15). As a consequence, the downstream effects and modifications identified in the EV cargo can be assigned specifically to the TGFBR2 expression status of MSI cells. For example, our previous studies showed that the reconstituted expression of TGFBR2
Table III. TGFBR2-regulated gene expression and the effect of doxycycline.

\begin{tabular}{llcc}
\hline \multicolumn{3}{c}{$\begin{array}{c}\text { HCT116-TGFBR2 } \\
\text { (FC) }\end{array}$} & \\
\cline { 2 - 3 } miRNA & NGS & RT-qPCR & $\begin{array}{c}\text { Control (FC) } \\
\text { RT-qPCR }\end{array}$ \\
\hline $\begin{array}{l}\text { Parental MSI cells } \\
\text { miR-381-3p }\end{array}$ & 0.33 & 0.39 & 0.99 \\
miR-379-5p & 0.35 & 0.39 & 1.04 \\
miR-181a-2-3p & 0.61 & 0.80 & 1.04 \\
EVs & & & \\
miR-381-3p & 0.50 & 0.55 & 1.05 \\
miR-376a-3p & 0.44 & 0.59 & 1.00 \\
\hline
\end{tabular}

FC values (TGFBR2 deficient/TGFBR2 proficient) represent mean values calculated from four biological replicates. Control values were obtained from HCT116-Tet-On cells. miR, microRNA; FC, fold change; TGFBR2, transforming growth factor- $\beta$ receptor type 2; MSI, microsatellite instability; EVs, extracellular vesicles; NGS, next-generation sequencing; RT-qPCR, reverse transcription-quantitative polymerase chain reaction.

alters the glycophenotype of MSI tumor cells (15,32,33), and tumor driver-induced glycome changes have been linked to TGFBR2-regulated genes (34). Additionally, the reconstituted expression of TGFBR2 can cause differential de novo protein expression (35). In addition to these (glyco)proteomic changes of MSI cells, the reconstituted expression of TGFBR2 can modulate the protein cargo of MSI-derived small EVs (14). Although TGFBR2 serves a crucial role in the MSI pathway of CRC development and progression, knowledge of the TGFBR2-dependent effects on the molecular cargo of small EVs released by MSI tumor cells remains limited. The present study provides evidence for TGFBR2-regulated miRNA expression profiles in EVs and corresponding parental cells that exhibit the MSI phenotype. Although gene expression differences between CRC and normal tissues and CRC cells and their EVs have been identified $(36,37)$, the contribution of TGFBR 2 to the expression patterns of non-coding RNA in MSI tumor cells and shed vesicles has not been investigated previously. Using high-throughput small RNA-Seq and the well-characterized HCT116-TGFBR2 model system, the present study identified 25 candidate miRNAs (cells $n=15$; EVs $\mathrm{n}=10$ ) for which differential expression appears to be regulated in a TGFBR2-dependent manner. As TGFBR2 deficiency is a hallmark of the majority of primary MSI tumors and is considered to be a MSI driver, those miRNAs with altered expression upon TGFBR 2 deficiency were of interest. In total, $56 \%$ of the miRNAs were found to be expressed in all four groups. At a qualitative level, this finding emphasizes the general overlap of miRNA profiles between MSI CRC cells and their secreted EVs but also indicated TGFBR2-dependent differences among these groups. More refined and quantitative analysis by DESeq 2 then provided additional evidence for the impact of a single tumor driver mutation on altering cellular and EV miRNA profiles. In particular, TGFBR2 deficiency 

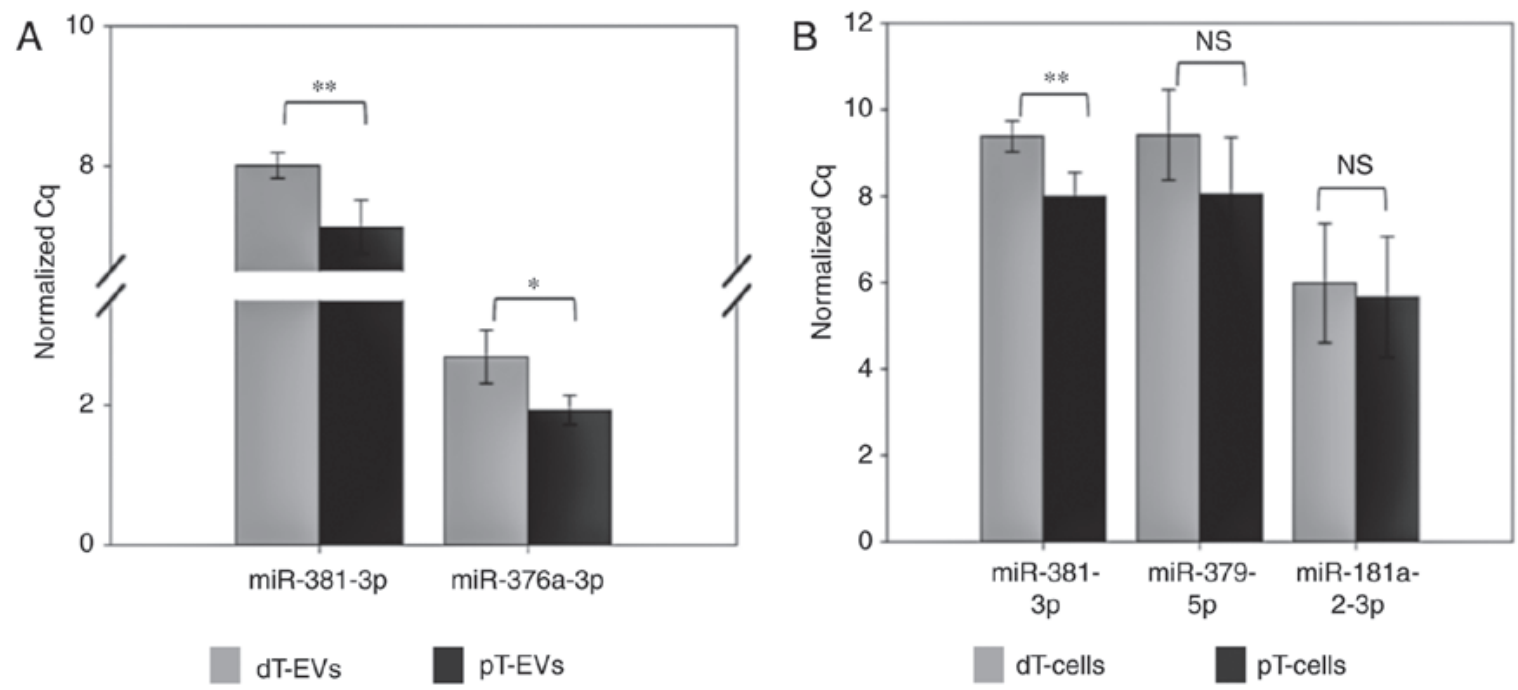

Figure 6. Validation of TGFBR2-dependent miRNAs. (A) Normalized Cq values of miR-381-3p were significantly lower (P=0.006) in pT EVs (dark grey) compared with those in dT EVs (light grey). Normalized Cq values of miR-376a-3p were significantly lower $(\mathrm{P}=0.013)$ in $\mathrm{pT}$ EVs compared with those in dT EVs. (B) Normalized Cq values of miR-381-3p were also significantly lower $(\mathrm{P}=0.005)$ in $\mathrm{pT}$ cells (dark grey) compared with those in dT cells (light grey). Expression analysis of miR-379-5p and miR-181a-2-3p revealed a trend towards lower expression in dT cells but did not reach statistical significance. All data are presented as mean values obtained from four biological replicates. "P $\mathrm{P} 0.05 ;{ }^{* *} \mathrm{P}<0.01$. miRNA/miR, microRNA; TGFBR2, transforming growth factor- $\beta$ receptor type 2; EVs, extracellular vesicles; dT, TGFBR2 deficient; pT, TGFBR2 proficient; Cq, quantification cycle; NS, not significant.

caused the upregulation and downregulation of five and 20 miRNAs, respectively. In independent RT-qPCR experiments, the differential expression of four TGFBR2-regulated miRNAs (miR-381-3p, miR-376a-3p, miR-379-5p and miR-181a-2-3p) was successfully validated. One of the validated candidates, miR-381-3p was downregulated in both dT EVs and dT cells. Accordingly, it was predicted that TGFBR2 deficiency leads to the downregulation of miR-381-3p in vivo. Such altered expression levels are expected to be of functional relevance and may contribute to MSI-specific tumor characteristics.

Several studies have shown that miR-381-3p can play a dual role in carcinogenesis. Depending on the cancer entity, it can confer tumor-promoting or suppressive effects $(38,39)$. For CRC, its expression was reported to be significantly downregulated in primary tumor tissue compared with that in the normal colon mucosa $(36,40)$. As reported by $\mathrm{Hu}$ et al, miR-381-3p is the only miRNA that targets the 3 -untranslated region of both Twist and Snail mRNA in the colon epithelium (41). TGFBR2-mediated TGF- $\beta$ signaling can trigger the expression of Twist and Snail and thereby facilitate the conversion of epithelial cells into motile mesenchymal cells $(42,43)$. As miR-381-3p has an inhibitory effect on epithelial-to-mesenchymal transition (EMT) through targeting Twist and Snail in colon cells, it is predicted that MSI tumors may gain invasive properties by downregulating miR-381-3p. In agreement with this hypothesis, MSI tumors typically invade the local tumor microenvironment (TME), have a lower propensity to metastasize to distant tissues and have a more favorable prognosis compared with microsatellite stable (MSS) phenotypes (44). Furthermore, liver metastases and poor survival rates of patients with CRC have been linked to elevated expression levels of miR-181a-2-3p $(45,46)$, which is a direct target of the TGF- $\beta$ pathway (47). This is in line with the data obtained in the present study demonstrating elevated expression levels of miR-181a-2-3p in pT cells and decreased expression levels in dT cells. Functional studies have shown that the restoration of miR-381-3p hindered the migratory and invasive capacity and proliferation of CRC cells by directly targeting LRH-1 and Twist-1 (36,40). Twist-1 can also be repressed by miR-134-5p (48), another candidate downregulated in dT cells. It was described that the overexpression of miR-134-5p resulted in repression of the proliferation and growth of CRC cells (49). Our previous study reported that the reconstitution of TGFBR2 in HCT116-TGFBR2 cells significantly reduced cellular proliferation (50). Based on the evidence mentioned above, this decreased proliferation rate in pT cells may at least partly be caused by elevated expression levels of miR-381-3p and miR-134-5p $(36,40,49)$. However, the possibility that multiple factors account for the altered proliferation rate cannot be excluded.

In gastric cancer, miR-381-3p and its target TMEM16A has been shown to contribute to cell invasion by promoting TGF- $\beta$ secretion $(51,52)$. TGF- $\beta$ is a potent inducer of EMT and thus serves a crucial role in cancer invasion, motility and metastasis (53). miR-381-3p suppressed TGF- $\beta$ signaling and EMT moderately by targeting TMEM16A.In this context, TGF- $\beta$ may operate as a mediator between miR-381-3p,TMEM16A and EMT (52). The process of EMT requires the tight control of cell junctions. It was reported that the tight junction proteins occludin and zonula occludens (ZO-1) are significantly higher expressed when miR-381-3p is downregulated in intestinal epithelial cells (54). This is in agreement with our previous proteomic study, which showed that ZO-1 was exclusively expressed in dT EVs, which exhibited significantly lower expression levels of miR-381-3p (14). This correlation suggests a functional role of miR-381-3p in EVs, which may contribute to the modulation of the TME by altering tight junctions at local and more distant sites. Although emerging evidence described the expression and functional relevance of miR-381-3p in various tissues, its expression and biological role in secreted EVs remains to be 
fully elucidated. Among other miRNA candidates, increased levels of miR-381-3p have been detected in small EVs isolated from TGF- $\beta 1$-stimlutated A549 human lung cancer cells with a mesenchymal phenotype, compared with small EVs isolated from untreated epithelial A549 cells or 16HBE human normal bronchial epithelial cells (55). In a previous study focusing on CRC, EVs were isolated from a cohort of 100 patients with CRC ( $n=25$ each of stages I-IV) and 50 healthy controls, and the EV-RNA content was compared between the two groups (56). Based on RNA-Seq data, it was reported that the number of CRC-associated miRNA isoforms (isomiRs) detected in EVs isolated from patients with CRC increased significantly with disease progression. Additionally, isoforms of miR-381-3p were identified in the cargo of EVs with significant differences between disease stages (56). In a follow up study, miR-381-3p and two other TGFBR2-regulated candidates (miR-376a-3p and miR-320d) were found to be downregulated in EVs isolated from patients with stage I and II CRC compared with the expression levels detected in EVs from healthy donors (57). In the present study, the downregulation of miR-381-3p and miR-376a-3p was detected in dTEVs, whereas higher expression levels of miR-320d were detected in dT EVs. Similarly, elevated expression levels of miR-320c were observed in dT EVs. Wang et al reported that miR-320c was significantly upregulated in plasma EVs from patients with early stage CRC (57). However, they did not include information regarding the MSS and MSI status of the CRC patient cohort, making it impossible to connect the observed changes in expression to the MSI phenotype or lack of TGFBR2 expression. In addition to miR-320c and miR-320d, the present study detected increased expression levels of miR-3187-3p in dT EVs. Experimental evidence suggests that miR-3178-3p is specifically sorted into CRC-derived EVs, leading to elevated levels compared with cellular miR-3178-3p expression (37). Further evidence has shown that increased expression levels of miR-3178-3p in the colonic epithelium can be caused by single nucleotide polymorphisms affecting the TGFBR1 gene (58). In the present study, an association between miR-3178-3p and TGFBR2 expression status was found. However, the function of miR-3178-3p in the pathogenesis of CRC remains to be elucidated.

By comparing miRNA expression levels between dT cells and pT cells, two miRNAs (miR-4521 and miR-31-3p) were upregulated in dT cells. It has been reported that miR-4521 is significantly upregulated in colon cancer stem cells compared with that in non-stem cells (59). Of note, TGF- $\beta$ signaling has emerged as key pathway for controlling stem cell renewal and the differentiation of intestinal epithelial cells (60). miR-4521-3p was also upregulated in patients with inflamed colonic epithelium of colitis ulcerosa (61). MSI colorectal tumors are highly immunogenic tumors and are often characterized by marked lymphocyte infiltration and inflammatory reactions (44). Therefore, it was hypothesized that high levels of miR-4521-3p may favor the inflammatory environment often observed in MSI tumors. The second candidate found to be upregulated in dT cells, miR-31-3p, has also been associated with intestinal inflammation (62). Olaru et al suggested that the expression of miR-31-3p increases during the progression of inflammation-associated intestinal neoplasia (63). Experiments have shown that miR-31-3p can repress the transcription factor E2F2 that controls the expression of several TGF- $\beta$ target genes, including survivin, c-myc and cyclin A2 (64). Using an miRNA array, Nosho et al reported higher expression levels of miR-31-3p/-5p in BRAF (V600E)-mutated CRC compared with $B R A F$ wild-type CRC (65). It was suggested that high expression levels of miR-31 are associated with the MSI phenotype, although they did not investigate the expression status of TGFBR2. In the present study, it was shown for the first time, to the best of our knowledge, that the upregulation of miR-31-3p may positively correlate with TGFBR2 deficiency in MSI tumor cells. In addition to being a potential MSI marker, other studies have highlighted the potential of miR-31-3p as a predictive biomarker of anti-epidermal growth factor receptor therapy efficacy for patients with $R A S$ wild-type and metastatic CRC (66).

In conclusion, the present study linked the regulation of 25 miRNAs in EVs and their MSI tumor cells to the expression status of TGFBR2. Although the study did not investigate which intracellular pathways downstream of TGFBR2 may affect the differential miRNA expression, specific tumor driver-dependent alterations in miRNA expression were identified in EVs and parental MSI tumor cells by combining a well-defined dox-inducible model system, a reproducible EV isolation protocol and high-throughput small RNA-Seq. In general, the results emphasize the broad overlap of miRNA content in EVs and their secreting cells. In particular, this study highlights the impact of the recurrent MSI tumor driver mutation affecting TGFBR2 on altering the miRNA signatures of EVs and their parental MSI CRC cells.

Future investigations are required to determine which intracellular pathways downstream of TGFBR2 caused the observed differential miRNA expression patterns. Although the TGF-/dox-mediated activation of canonical signaling was shown in the model system, ligand binding can also stimulate several non-canonical pathways downstream of the receptor that may have an impact on miRNA regulation. This can be addressed in further experiments by selectively targeting each of these different pathways using specific inhibitors. In addition, the results of the present study require confirmation in additional MSI CRC cell lines and the role of miRNA candidates in MSI tumorigenesis requires assessment though functional studies.

\section{Acknowledgements}

The authors gratefully acknowledge Mrs. Ulrike Ganserer (EM-Lab, University Hospital Heidelberg), Mrs. Sigrun Himmelsbach and Mrs. Vera Fuchs (Dept. of Applied Tumor Biology, University Hospital Heidelberg) for their technical assistance, Mr. Benedikt Kirchner (Dept. of Animal Physiology and Immunology, Technical University of Munich) and Dr Christine Wurmser (Animal Breeding, Technical University of Munich) for their support in sequencing and processing small RNA libraries, and Claussen-Simon-Stiftung for their encouragement.

\section{Funding}

This study was supported by intramural funding from the Technical University of Munich (MP) and the University 
Hospital Heidelberg (grant nos. F.204078 to JG and F.204008 to JK).

\section{Availability of data and materials}

Sequencing data were deposited in the European Nucleotide Archive (ENA) under accession no. PRJEB30305 (http://www. ebi.ac.uk/ena/data/view/PRJEB30305) (67). EV data were submitted to the EV-TRACK knowledgebase (http://evtrack. org/; EV track ID: EV180072) (68).

\section{Authors' contributions}

JG, JK, MP, FF, VM and DB designed the experiments. FF, VM, IH performed the experiments. All authors contributed to data analysis. JG, JK, MP, FF, VM and DB prepared the manuscript. All authors have approved the final manuscript.

\section{Ethics approval and consent to participate}

Not applicable.

\section{Patient consent for publication}

Not applicable.

\section{Competing interests}

The authors declare that they have no competing interests.

\section{References}

1. Boland CR and Goel A: Microsatellite instability in colorectal cancer. Gastroenterology 138: 2073-2087.e3, 2010.

2. Woerner SM, Benner A, Sutter C, Sutter C, Schiller M, Yuan YP, Keller G, Bork P, Doeberitz Mv and Gebert JF: Pathogenesis of DNA repair-deficient cancers: A statistical meta-analysis of putative Real Common Target genes. Oncogene 22: 2226-2235, 2003.

3. Markowitz S, Wang J, Myeroff L, Parsons R, Sun L, Lutterbaugh J, Fan RS, Zborowska E, Kinzler KW, Vogelstein B, et al: Inactivation of the type II TGF-beta receptor in colon cancer cells with microsatellite instability. Science 268: 1336-1338, 1995.

4. Massagué J: TGF $\beta$ signalling in context. Nat Rev Mol Cell Biol 13: 616-630, 2012.

5. Butz H, Rácz K, Hunyady L and Patócs A: Crosstalk between TGF- $\beta$ signaling and the microRNA machinery. Trends Pharmacol Sci 33: 382-393, 2012.

6. Bartel DP: MicroRNAs: Genomics, biogenesis, mechanism, and function. Cell 116: 281-297, 2004.

7. Gebert LFR and MacRae IJ: Regulation of microRNA function in animals. Nat Rev Mol Cell Biol 20: 21-37, 2019.

8. Friedman RC, Farh KK, Burge CB and Bartel DP: Most mammalian mRNAs are conserved targets of microRNAs. Genome Res 19: 92-105, 2009.

9. Zhang J, Li S, Li L, Li M, Guo C, Yao J and Mi S: Exosome and exosomal microRNA: Trafficking, sorting, and function. Genomics Proteomics Bioinformatics 13: 17-24, 2015.

10. Cantini L, Isella C, Petti C, Picco G, Chiola S, Ficarra E, Caselle $\mathrm{M}$ and Medico E: MicroRNA-mRNA interactions underlying colorectal cancer molecular subtypes. Nat Commun 6: 8878,2015

11. van Niel G, D'Angelo G and Raposo G: Shedding light on the cell biology of extracellular vesicles. Nat Rev Mol Cell Biol 19: 213-228, 2018.

12. Bellingham SA, Shambrook $M$ and Hill AF: Quantitative Analysis of Exosomal miRNA via qPCR and Digital PCR. Methods Mol Biol 1545: 55-70, 2017.
13. Mathieu M, Martin-Jaular L, Lavieu G and Théry C: Specificities of secretion and uptake of exosomes and other extracellular vesicles for cell-to-cell communication. Nat Cell Biol 21: 9-17, 2019.

14. Fricke F, Lee J, Michalak M, Warnken U, Hausser I, Suarez-Carmona M, Halama N, Schnölzer M, Kopitz J and Gebert J: TGFBR2-dependent alterations of exosomal cargo and functions in DNA mismatch repair-deficient HCT116 colorectal cancer cells. J Cell Commun Signal 15: 14, 2017.

15. Lee J, Ballikaya S, Schönig K, Ball CR, Glimm H, Kopitz J and Gebert J: Transforming growth factor beta receptor 2 (TGFBR2) changes sialylation in the microsatellite unstable (MSI) Colorectal cancer cell line HCT116. PLoS One 8: e57074, 2013.

16. Welman A, Barraclough J and Dive C: Generation of cells expressing improved doxycycline-regulated reverse transcriptional transactivator rtTA2S-M2. Nat Protoc 1: 803-811, 2006.

17. Andrews S: FastQC: A quality control tool for high throughput sequence data. Bioinformatics Babraham. http://www.bioinformatics.babraham.ac.uk/projects/fastqc. Accessed 18 Dec, 2018.

18. Kong Y: Btrim: A fast, lightweight adapter and quality trimming program for next-generation sequencing technologies. Genomics 98: 152-153, 2011.

19. The RNAcentral Consortium; Petrov AI, Kay SJE, Kalvari I, Howe KL, Gray KA, Bruford EA, Kersey PJ, Cochrane G, Finn RD, et al: A comprehensive database of non-coding RNA sequences. Nucleic Acids Res 45: D28-D134, 2017.

20. Kozomara A and Griffiths-Jones S: miRBase: Annotating high confidence microRNAs using deep sequencing data. Nucleic Acids Res 42: 68-73, 2014.

21. Langmead B, Trapnell C, Pop M and Salzberg SL: Ultrafast and memory-efficient alignment of short DNA sequences to the human genome. Genome Biol 10: R25, 2009.

22. Love MI, Huber W and Anders S: Moderated estimation of fold change and dispersion for RNA-seq data with DESeq2. Genome Biol 15: 550, 2014.

23. Benjamini Y and Hochberg Y: Controlling the False discovery rate: A practical and powerful approach to multiple testing. J R Stat Soc Series B Stat Methodol 57: 289-300, 1995.

24. Fan Y, Siklenka K, Arora SK, Ribeiro P, Kimmins S and Xia J: miRNet-dissecting miRNA-target interactions and functional associations through network-based visual analysis. Nucleic Acids Res 44: W135-W141, 2016.

25. Fabregat A, Sidiropoulos K, Garapati P, Gillespie M, Hausmann K, Haw R, Jassal B, Jupe S, Korninger F, McKay S, et al: The Reactome pathway Knowledgebase. Nucleic Acids Res 44: D481-D487, 2016.

26. Pfaffl MW: A new mathematical model for relative quantification in real-time RT-PCR. Nucleic Acids Res 29: e45, 2001.

27. Livak KJ and Schmittgen TD: Analysis of relative gene expression data using real-time quantitative PCR and the 2(-Delta Delta C(T)) method. Methods 25: 402-408, 2001.

28. Andersen CL, Jensen JL and Ørntoft TF: Normalization of real-time quantitative reverse transcription-PCR data: A model-based variance estimation approach to identify genes suited for normalization, applied to bladder and colon cancer data sets. Clin Cancer Res 64: 5245-5250, 2004.

29. Vandesompele J, De Preter K, Pattyn F, Poppe B, Van Roy N, De Paepe A and Speleman F: Accurate normalization of real-time quantitative RT-PCR data by geometric averaging of multiple internal control genes. Genome Biol 3: RESEARCH0034, 2002.

30. Théry C, Witwer KW, Aikawa E, Alcaraz MJ, Anderson JD, Andriantsitohaina R, Antoniou A, Arab T, Archer F, Atkin-Smith GK, et al: Minimal information for studies of extracellular vesicles 2018 (MISEV2018): A position statement of the International Society for Extracellular Vesicles and update of the MISEV2014 guidelines. J Extracell Vesicles 7: 1535750, 2018.

31. Ewing B, Hillier L, Wendl MC and Green P: Base-calling of automated sequencer traces using phred. I. Accuracy assessment. Genome Res 8: 175-185, 1998.

32. Patsos G, André S, Roeckel N, Gromes R, Gebert J, Kopitz J and Gabius HJ: Compensation of loss of protein function in microsatellite-unstable colon cancer cells (HCT116): A gene-dependent effect on the cell surface glycan profile. Glycobiology 19: 726-734, 2009.

33. Lee J, Warnken U, Schnölzer M, Gebert J and Kopitz J: A new method for detection of tumor driver-dependent changes of protein sialylation in a colon cancer cell line reveals nectin-3 as TGFBR2 target. Protein Sci 24: 1686-1694, 2015.

34. Lee J, Katzenmaier EM, Kopitz J and Gebert J: Reconstitution of TGFBR2 in HCT116 colorectal cancer cells causes increased LFNG expression and enhanced N-acetyl-d-glucosamine incorporation into Notch1. Cell Signal 28: 1105-1113, 2016. 
35. Lee J, Fricke F, Warnken U, Schnölzer M, Kopitz J and Gebert J: Reconstitution of TGFBR2-mediated signaling causes upregulation of GDF-15 in HCT116 colorectal cancer cells. PLoS One 10 e0131506, 2015

36. He X, Wei Y, Wang Y, Liu L, Wang W and Li N: MiR-381 functions as a tumor suppressor in colorectal cancer by targeting Twist1. Onco Targets Ther 9: 1231-1239, 2016.

37. Cha DJ, Franklin JL, Dou Y, Liu Q, Higginbotham JN, Demory Beckler M, Weaver AM, Vickers K, Prasad N, Levy S, et al: KRAS-dependent sorting of miRNA to exosomes. Elife 4: e07197, 2015.

38. Tang H, Wang Z, Liu Q, Liu X, Wu M and Li G: Disturbing miR-182 and -381 inhibits BRD7 transcription and glioma growth by directly targeting LRRC4. PLoS One 9: e84146, 2014.

39. Zhang M, Huang S and Long D: MiR-381 inhibits migration and invasion in human gastric carcinoma through downregulatedting SOX4. Oncol Lett 14: 3760-3766, 2017.

40. Liang Y, Zhao Q, Fan L, Zhang Z, Tan B, Liu Y and Li Y: Down-regulation of MicroRNA-381 promotes cell proliferation and invasion in colon cancer through up-regulation of LRH-1. Biomed Pharmacother 75: 137-141, 2015.

41. Hu WW, Chen PC, Chen JM, Wu YM, Liu PY, Lu CH, Lin YF, Tang $\mathrm{CH}$ and Chao CC: Periostin promotes epithelial-mesenchymal transition via the MAPK/miR-381 axis in lung cancer. Oncotarget 8: 62248-62260, 2017.

42. Thiery JP, Acloque H, Huang RY and Nieto MA: Epithelial-mesenchymal transitions in development and disease. Cell 139: 871-890, 2009.

43. Heldin $\mathrm{CH}$, Vanlandewijck M and Moustakas A: Regulation of EMT by TGFß in cancer. FEBS Lett 586: 1959-1970, 2012.

44. Buckowitz A, Knaebel HP, Benner A, Bläker H, Gebert J, Kienle P, von Knebel Doeberitz M and Kloor M: Microsatellite instability in colorectal cancer is associated with local lymphocyte infiltration and low frequency of distant metastases. $\mathrm{Br}$ J Cancer 92: 1746-1753, 2005.

45. Nishimura J, Handa R, Yamamoto H, Tanaka F, Shibata K, Mimori K, Takemasa I, Mizushima T, Ikeda M, Sekimoto M, et al: microRNA-181a is associated with poor prognosis of colorectal cancer. Oncol Rep 28: 2221-2226, 2012.

46. Ji D, Chen Z, Li M, Zhan T, Yao Y, Zhang Z, Xi J, Yan L and Gu J: MicroRNA-181a promotes tumor growth and liver metastasis in colorectal cancer by targeting the tumor suppressor WIF-1. Mol Cancer 13: 86, 2014

47. Taylor MA, Sossey-Alaoui K, Thompson CL, Danielpour D and Schiemann WP: TGF- $\beta$ upregulates miR-181a expression to promote breast cancer metastasis. J Clin Invest 123: 150-163, 2013.

48. Ji LJ, Su J, Xu AL, Pang B and Huang QM: MiR-134-5p attenuates neuropathic pain progression through targeting Twist1. J Cell Biochem: Sep 6, 2018 doi: 10.1002/jcb.27486 (Epub ahead of print).

49. El-Daly SM, Abba ML, Patil N and Allgayer H: miRs-134 and -370 function as tumor suppressors in colorectal cancer by independently suppressing EGFR and PI3K signalling. Sci Rep 6: 24720, 2016

50. Oh BY, Kim SY, Lee YS, Hong HK, Kim TW, Kim SH, Lee WY and Cho YB: Twist1-induced epithelial-mesenchymal transition according to microsatellite instability status in colon cancer cells. Oncotarget 7: 57066-57076, 2016

51. Liu F, Cao QH, Lu DJ, Luo B, Lu XF, Luo RC and Wang XG: TMEM16A overexpression contributes to tumor invasion and poor prognosis of human gastric cancer through TGF- $\beta$ signaling. Oncotarget 6: 11585-11599, 2015.

52. Cao Q, Liu F, Ji K, Liu N, He Y,Zhang W and Wang L: MicroRNA381 inhibits the metastasis of gastric cancer by targeting TMEM16A expression. J Exp Clin Cancer Res 36: 29, 2017.
53. Katsuno Y, Lamouille S and Derynck R: TGF- $\beta$ signaling and epithelial-mesenchymal transition in cancer progression. Curr Opin Oncol 25: 76-84, 2013.

54. Liu L, Yao J, Li Z, Zu G, Feng D, Li Y, Qasim W, Zhang S, Li T, Zeng $\mathrm{H}$ and Tian X: miR-381-3p knockdown improves intestinal epithelial proliferation and barrier function after intestinal ischemia/reperfusion injury by targeting nurr1. Cell Death Dis 9: 411, 2018.

55. Tang YT, Huang YY, Li JH, Qin SH, Xu Y, An TX, Liu CC, Wang $Q$ and Zheng L: Alterations in exosomal miRNA profile upon epithelial-mesenchymal transition in human lung cancer cell lines. BMC Genomics 19: 802, 2018.

56. Yuan T, Huang X, Woodcock M, Du M, Dittmar R, Wang Y, Tsai S, Kohli M, Boardman L, Patel T and Wang L: Plasma extracellular RNA profiles in healthy and cancer patients. Sci Rep 6: 19413, 2016.

57. Wang J, Yan F, Zhao Q, Zhan F, Wang R, Wang L, Zhang Y and Huang X: Circulating exosomal miR-125a-3p as a novel biomarker for early-stage colon cancer. Sci Rep 7: 4150, 2017.

58. Slattery ML, Trivellas A, Pellatt AJ, Mullany LE, Stevens JR, Wolff RK and Herrick JS: Genetic variants in the TGF $\beta$-signaling pathway influence expression of miRNAs in colon and rectal normal mucosa and tumor tissue. Oncotarget 8: 16765-16783, 2017.

59. Fang Y, Xiang J, Chen Z, Gu X, Li Z, Tang F and Zhou Z: miRNA expression profile of colon cancer stem cells compared to non-stem cells using the SW1116 cell line. Oncol Rep 28: 2115-2124, 2012.

60. Mishra L, Derynck R and Mishra B: Transforming growth factor-beta signaling in stem cells and cancer. Science 310: 68-71, 2005.

61. Valmiki S, Ahuja V and Paul J: MicroRNA exhibit altered expression in the inflamed colonic mucosa of ulcerative colitis patients. World J Gastroenterol 23: 5324-5332, 2017.

62. Fang K, Law IKM, Padua D, Sideri A, Huang V, Kevil CG, Iliopoulos D and Pothoulakis C: MicroRNA-31-3p is involved in substance $\mathrm{P}(\mathrm{SP})$-associated inflammation in human colonic epithelial cells and experimental colitis. Am J Pathol 188: 586-599, 2018.

63. Olaru AV, Selaru FM, Mori Y, Vazquez C, David S, Paun B, Cheng Y, Jin Z, Yang J, Agarwal R, et al: Dynamic changes in the expression of MicroRNA-31 during inflammatory bowel disease-associated neoplastic transformation. Inflamm Bowel Dis 17: 221-231, 2011.

64. Li T, Luo W, Liu K, Lv X and Xi T: miR-31 promotes proliferation of colon cancer cells by targeting E2F2. Biotechnol Lett 37 : 523-532, 2015.

65. Nosho K, Igarashi H, Nojima M, Ito M, Maruyama R, Yoshii S, Naito T, Sukawa Y, Mikami M, Sumioka W, et al: Association of microRNA-31 with BRAF mutation, colorectal cancer survival and serrated pathway. Carcinogenesis 35: 776-783, 2014

66. Laurent-Puig P, Grisoni ML, Heinemann V, Liebaert F, Neureiter D, Jung A, Montestruc F, Gaston-Mathe Y, Thiébaut R and Stintzing S: Validation of miR-31-3p expression to predict cetuximab efficacy when used as first-line treatment in RAS Wild-type metastatic colorectal cancer. Clin Cancer Res 25 134-141, 2019.

67. Harrison PW, Alako B, Amid C, Cerdeño-Tárraga A, Cleland I, Holt S, Hussein A, Jayathilaka S, Kay S, Keane T, et al: The European nucleotide archive in 2018. Nucleic Acids Res 47: D84-D88, 2019.

68. EV-TRACK Consortium; Van Deun J, Mestdagh P, Agostinis P, Akay Ö, Anand S, Anckaert J, Martinez ZA, Baetens T, Beghein E, et al: EV-TRACK: Transparent reporting and centralizing knowledge in extracellular vesicle research. Nat Methods 14: 228-232, 2017 